\title{
Schwannoma of the hypoglossal nerve: Review of the literature based on an illustrative case
}

\author{
ROSARIO FORNARO, ALEXANDER SALERNO, DAVID CONSTANTIN FILIP, \\ ELISA CARATTO, MICHELA CARATTO and MARCO CASACCIA
}

Department of Surgery, IRCCS-Azienda Ospedaliera Universitaria San Martino-IST, University of Genoa, I-16132 Genoa, Italy

Received November 9, 2016; Accepted March 16, 2017

DOI: $10.3892 / \operatorname{mco} .2017 .1297$

\begin{abstract}
Schwannomas are benign tumours that originate from the myelin sheath of peripheral nerves. They are characterised by a slow growth tendency. Benign schwannomas represent $35 \%$ of the head and neck district tumours. Hypoglossal schwannomas account for $5 \%$ of non-vestibular schwannomas, and malignant schwannomas occur very rarely. In the present case report, the case of a 49-year-old man who presented with paraesthesias in the left parotid and submandibular region, associated with sensation of foreign bodies and dysphagia for solids, is described. A clinical examination revealed the presence of an ovoid palpable mass in the lateral-cervical region of the neck. The patient subsequently underwent excisional surgery, and neuropathological evaluation of the specimen confirmed the diagnosis of benign schwannoma with Antoni areas A and B. Despite the rarity of schwannomas, this condition should be considered in differential diagnoses for masses localised in the neck, as in cases where they reach considerable sizes ( $>3 \mathrm{~cm}$ in diameter). Surgery therefore represents the first-choice treatment.
\end{abstract}

\section{Introduction}

The hypoglossal nerve $(\mathrm{HyN})$ is a somatic nerve that innervates the muscles of the tongue and provides motor fibres derived from the cervical plexus to the muscles of the upper and lower hyoid region (1). Whereas tumours of the peripheral nerves are relatively infrequent, tumours of the $\mathrm{HyN}$ must be considered a rare event, accounting for only $5 \%$ of non-vestibular schwannomas (2,3). As reported in Table I, a review of literature on Medline for the words 'hypoglossal' and 'schwannoma' revealed only a low number of papers that have been currently published on this topic.

Correspondence to: Professor Rosario Fornaro, Department of Surgery, IRCCS-Azienda Ospedaliera Universitaria San Martino-IST, University of Genoa, Largo Rosanna Benzi 10, I-16132 Genoa, Italy

E-mail: rfornaro@unige.it

Key words: schwannoma, neurilemmoma, surgery, neck, tumour, report, review
The HyN (XII pair) originates from the hypoglossal nucleus that is located in the medulla oblongata. The efferent fibres, which are only somatic motor fibres, innervate the posterior belly of the digastric muscle, the sternohyoid muscle, the omohyoid muscle, the thyrohyoid muscle and the muscles of the tongue, such as the styloglossus, the genioglossus and the hyoglossus muscles. The fibres emerge from the anterolateral sulcus of the bulb as a longitudinal series of rootlets that then fuse together, to give origin to the nerve. After its short, although clinically relevant, intracranial (IC) course, the nerve exits, passing through the hypoglossal canal. Once extracranial (EC), the nerve is located in the parapharyngeal space (3). It descends first posteriorly, and then between the neurovascular bundle (internal carotid, phrenic nerve and internal jugular vein) directed towards the base of the tongue. During its course, it gives various collateral branches to the muscles of the hyoid region. Once it reaches the submandibular lodge, it divides into its terminal branches that finally innervate the muscles of the tongue $(1,4)$.

Schwannomas were described for the first time by Verocay in $1910(5,6)$. They are included in a more extended group of tumours of the peripheral nerve, which have been classically distinguished by Virchow in terms of real neuromas (traumatic neuromas and ganglioneuromas) and false neuromas (neurofibromas and neurilennomas) (5-7). They may be distinguished as being benign or malign, according to their biological behaviour.

\section{Case report}

History and examination. In the present report, the case is described of a 49-year-old male patient who, for the past year, had reported paraesthesias in the left parotid and submandibular region, associated for the previous 20 days with the sensation of a foreign body in the ipsilateral latero-cervical region and dysphagia for solids. A clinical examination revealed the presence of an ovoid palpable mass (5-6 cm in diameter) of hard elastic consistency that was mobile compared with the superficial plans, but adherent compared with the deep ones, neither painful nor tender.

Diagnostic testing and imaging. Ultrasonography of the neck revealed a hypoechoic lesion of solid consistency of $\sim 6 \mathrm{~cm}$ in diameter. It was adjacent to the major vessels of the neck, 
localised deeply and posteriorly relative to the submandibular gland. Magnetic resonance imaging (MRI) demonstrated a tendency to outward dislocation of the major vessels of the neck, the intense vascularization of the lesion and the remarkable mass effect on the lateral walls of the hypopharynx.

Surgery. After having obtained the informed consent, the patient underwent surgical exeresis of the mass, which appeared to be a voluminous ovoid lesion, laid deep on a plane between the lateral wall of the pharynx-hypopharynx (medially) and the neurovascular bundle of the neck, the stylohyoid muscle and the posterior face of the digastric muscle (laterally).

Originating from the HyN (XII pair), surgery started with an incision along the anterior margin of the sternocleidomastoid muscle, with the neck of the patient hyperextended and rotated contralaterally. Once the platysma muscle had been incised, the anterior margin of the sternocleidomastoid muscle was isolated, together with the interior jugular vein that was in direct contact with the medial surface of the muscle. This manoeuvre exposed the venous thyrolinguofacial trunk, also termed Farabeuf's trunk. The sectioning of such a venous trunk provided the opportunity to reach a deeper plane, where it was possible to identify and isolate the HyN. After the careful isolation of the carotid, of the carotidal bifurcation and of the phrenic nerve that spared the carotid glomus to prevent cardiovascular effects, the HyN was exposed superiorly. The lesion was located deep in the superior part of the nerve. Due to the location of the lesion, an accurate and fine isolation was surgically required. In order to accomplish this, the nerve from the surrounding connections with the carotids, the small vessels supplying the nerve, and the connections with the phrenic nerve were detached. Caution was required during this part of the operation, since an accidental lesion of the HyN could cause ipsilateral deviation of the tongue, peripheral dysarthria and chewing disorders. After all the previous structures had been gently displaced, the neoplasm was exposed, starting from its external surface. A good cleavage plane was present, and therefore it was possible to easily detach the mass anteriorly, posteriorly and inferiorly. There only appeared to be only one point of intimate contiguity between the mass and the nearby glossopharyngeal nerve. Once this has been isolated from the nervous structure, a further manoeuvre granted the dissection from the pharyngeal wall. In this manner, the mass was completely separated from the surrounding tissues, and could be finally excised.

Histopathological examination. Macroscopical examination of the specimen revealed a capsulated mass, with haemorrhagic areas to the cut. Microscopically, the lesion was formed by fused elements, occasionally structured in interlaced bundles. High cellular density (Antoni A areas) and low cellular density areas embedded in a connective matrix (Antoni B areas) were revealed. This evidence, in addition to the identified immunohistochemical positivity to S-100 protein, established the diagnosis of a benign schwannoma with Antoni A and B areas (8).

Follow-up. Neither signs of local recurrence nor of postoperative complications were apparent after 24 months, confirming the absence of residual masses. However, the patient reported the persistence of a moderate lowering of the voice, presumably caused by the impairment of the superior laryngeal nerve that had not been resolved by surgery.

\section{Discussion}

Clinical features. A specific diagnosis of schwannoma is difficult to make, and is frequently made at rather an advanced stage. Growth occurs slowly, and the onset of symptoms may only be determined after a long time period; the neurological signs and symptoms are, however, poor. Potentially useful criteria to identify schwannomas may be epidemiology and topography, considering that schwannomas are the most frequent neuronal tumours and that they represent $35 \%$ of the head and neck district tumours. The correct diagnosis of a schwannoma has important clinical and prognostic repercussions, as they may feature malignant characteristics, even if only in $2 \%$ of cases. On the clinical side, the prognosis is correlated with precocity of diagnosis and with the required radical surgical intervention. Clinically, these tumours tend to be asymptomatic, until they are of a small size $(<1-2 \mathrm{~cm}$ in diameter). Only when they reach certain dimensions $(\sim 2-3 \mathrm{~cm})$ may they be responsible for causing pain and paraesthesias; in time, they are able to increase their volume up to $4-5 \mathrm{~cm}$ (although rarely more than this), and, in these cases, they cause compression and dislocation without infiltration of the nerve of origin, with specific functional deficits that may be useful in terms of identifying the area and the nerve involved.

Location of the lesions. With regard to the location of the lesions, from an analysis of data specifically associated with hypoglossal schwannomas, it seems that, most frequently, these lesions have an IC localization, either developing in the cerebellopontine angle from the origin of the nerve or in the hypoglossal canal, and a secondary EC extension, most frequently in the parapharyngeal space. Only Yu et al (9) have reported on a patient who presumably developed a primary EC lesion that extended intracranially. As observed from a study of the literature, and as confirmed by Lee et al (2), the incidence of isolated EC lesions, such as occurred in the present case report, appears to be extremely rare.

Symptomatological heterogeneity. Depending on the dimensions of the lesion, the clinical picture may vary greatly. In almost all cases, there are varying degrees of paresis of the HyN, which appear as ipsilateral deviation, hemiatrophy and fasciculations of the tongue. These symptoms may be accompanied by hoarseness, intermittent-to-persistent headaches, dysarthria, slurred speech, swallowing difficulties and throat cramps $(2,4,9-28)$. Whereas the effects on the specific functions of the nerve may be attributable to the local damage of the tumour on the nerve, these types of lesions, particularly when they increase in volume, may determine mass effects on surrounding or adjacent structures; for example, Mariniello et al (19) reported on the case of a patient who exhibited signs of cerebellar ataxia, right-sided hypotonia and increased intracranial pressure. Another case that best clarifies the occurrence of mass effect is the patient reported by Li et al (18). In that case, there was an impairment of multiple cranial nerves and of the visual functions, as attested by MRI 


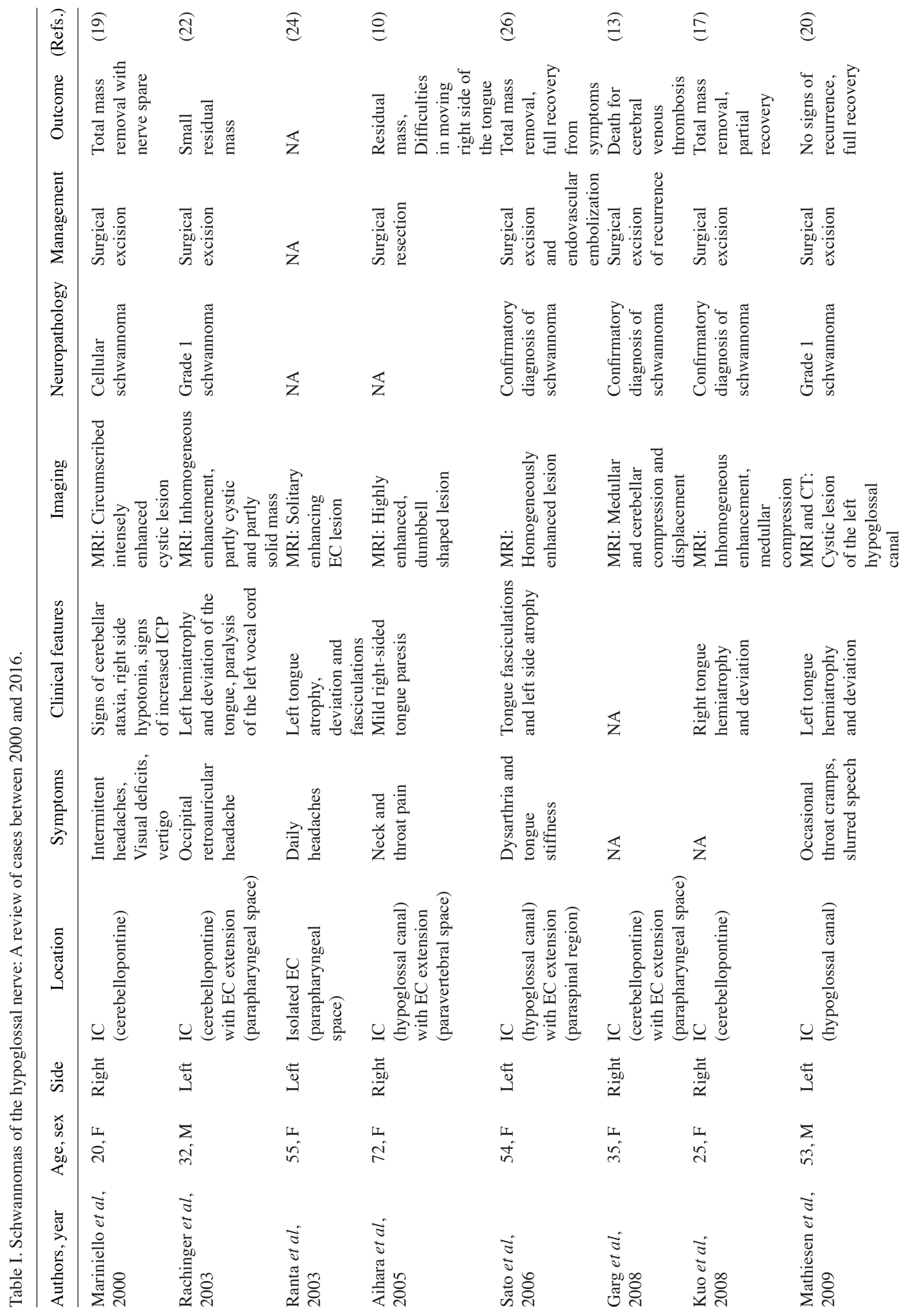




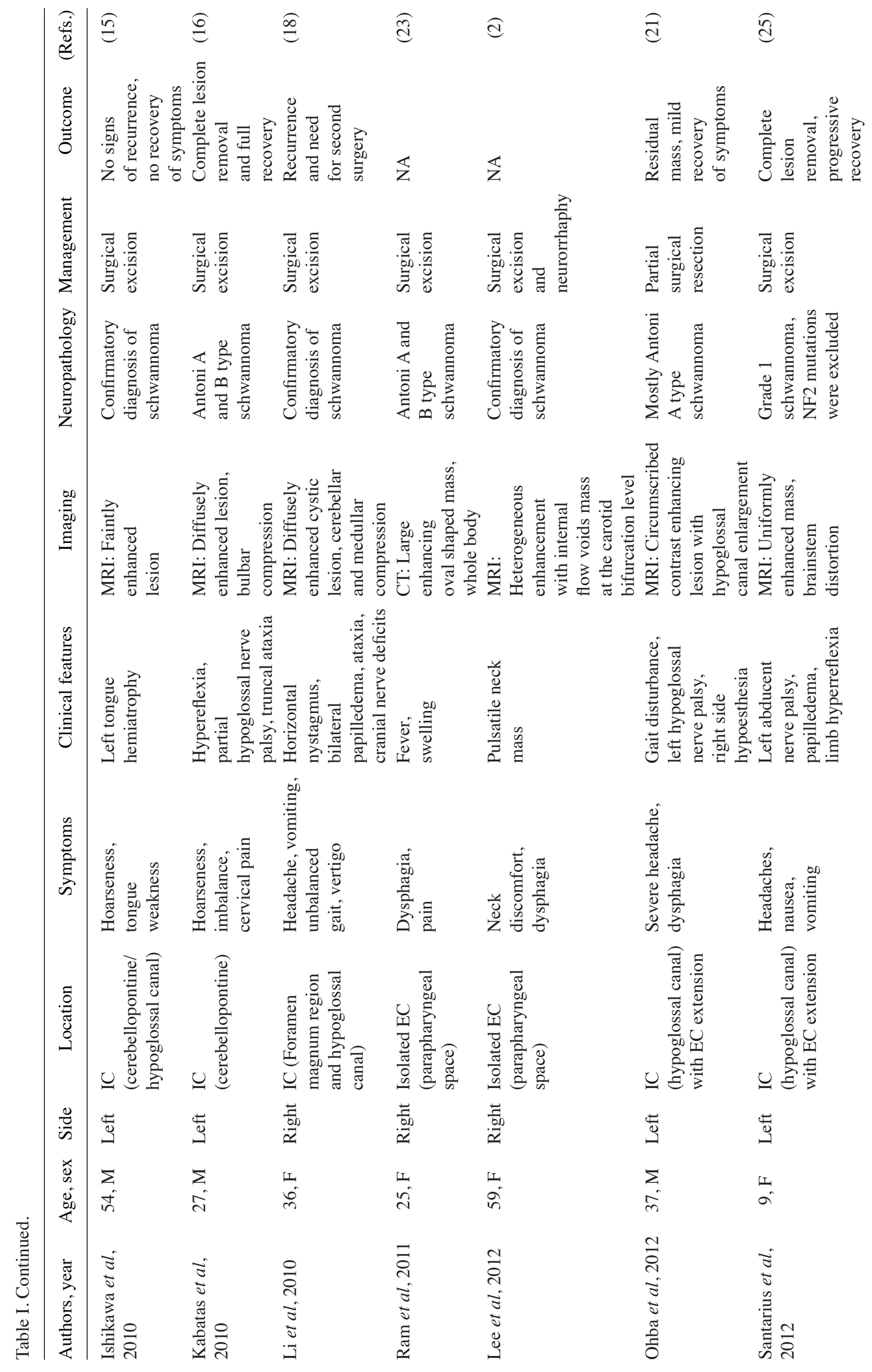




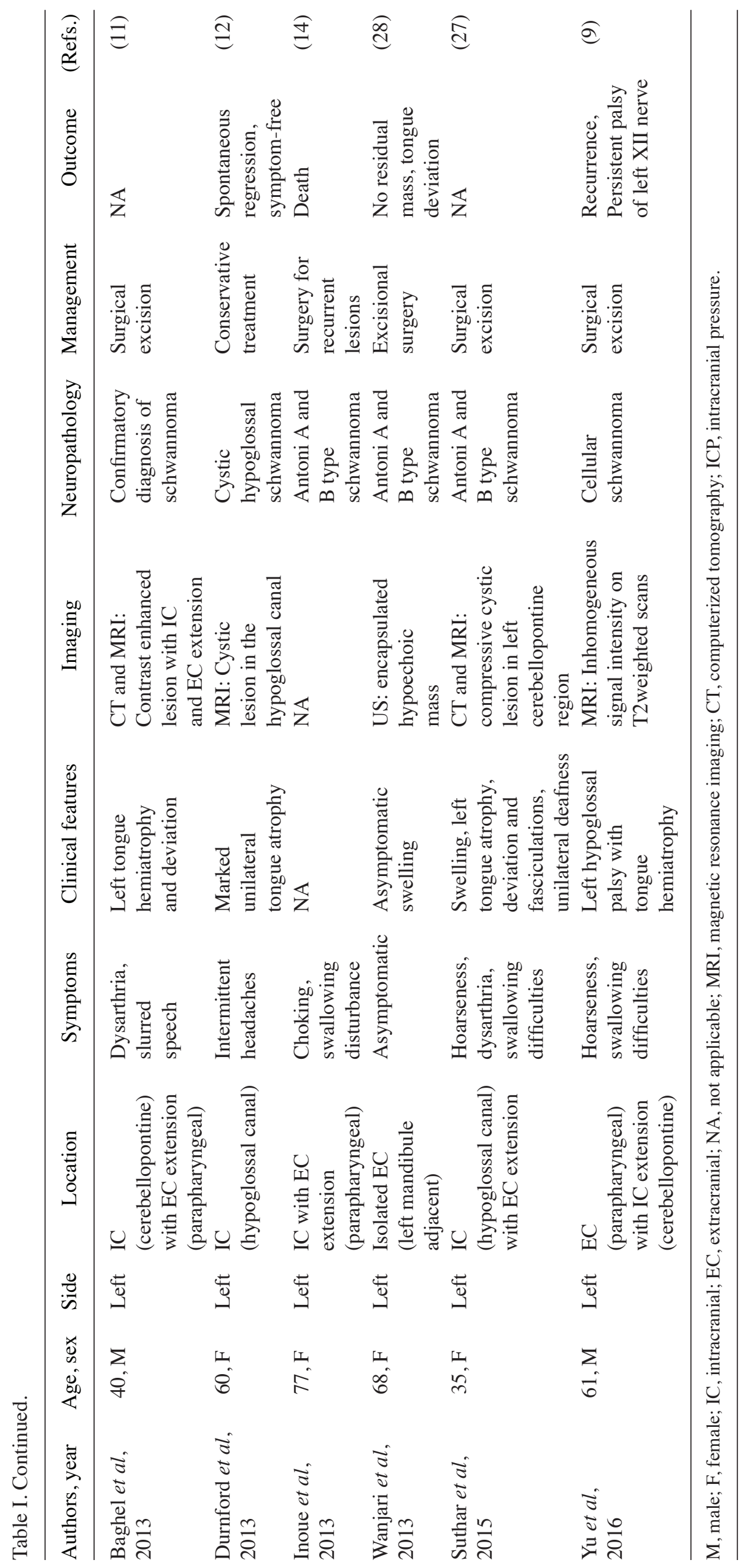


scanning, which provided evidence of cerebellar and medullar compression.

Imaging and diagnosis. To better define localization of the lesion and the involvement of adjacent structures, second-level imaging techniques, such as computed tomography and MRI, may be useful to the surgeon in order to define a correct preoperative plan (29). In fact, as this type of surgery is often characterised by technical difficulties, knowing the exact localization of the lesion and mastering the anatomy of the neck to overcome possible obstacles is of fundamental importance. In this manner, schwannomas may be completely excised without major problems or complications.

Treatment. Independently from the nerve that is affected, surgical treatment consists of systematic exeresis of the lesions. This has been furthermore confirmed by the experience of the present authors and by case reports $(2,9-28,30,31)$. In fact, all except one of the cases were treated surgically. Early treatment has important repercussions: First of all, even if a correct and refined clinical and imaging diagnosis is posed, it is impossible to make a definitive diagnosis without a histological examination of the excised specimen. In addition, as previously mentioned, these masses tend to grow slowly, inducing compressive effects on the surrounding structures. As a result, these could be dislocated and damaged, thus requiring a more delicate surgical procedure that may be burdened by a higher rate of complications. Furthermore, schwannomas are radioresistant, and therefore there are no obtainable benefits resulting from radiotherapeutic treatments. Damage and morbidity derived from lesions of the nerve from which the schwannoma originates may be prevented by accurately identifying and studying the nerve involved. As previously observed, the mass may be located in an eccentric position, well separated from the nerve fibres from a fibrotic capsule. This provides the surgeon with the possibility to remove the mass without generally impacting on the function of the nerve, if correctly identified. Even in the worst-case scenarios, however, damage to the nerve is mild. This is not true for malignant schwannomas, which are characterised by infiltrating behaviour towards the surrounding tissue. For this reason, in order to attempt complete removal of the lesion, the interruption or sacrifice of the nerve may be required. In these cases, in order to limit morbidity, reconstruction by direct nerve repair with epineurial microsutures or nervous grafts may be performed. In addition to these procedures, there are novel and upcoming techniques using biocompatible scaffolds that may help in the reconstruction of the transected nerve (32-36).

Neuropathological features. According to their biological behaviour, schwannomas may be distinguished in benign and malign lesions. Benign schwannomas are confined, and encapsulated within the epineurium. They appear as clearly defined masses in eccentric positions, either adherent to or detached from the nerve. Tumours usually create grey solid masses, but may also present as cystic areas with xanthomatous modifications. Histologically, two different forms may be distinguished according to growth features: Antoni form A is characterised by prolonged bundles of bipolar elements, associated with argyrophilic fibrils with nuclei aligned in parallel termed 'palisades', separated by hyaline bands, also termed Verocay bodies (8,37-40); Antoni form B is characterised by polymorphic and frequently astrocyte-like elements, associated with mucus and microcystic degeneration (8,37-40). There may be degenerative phenomena, such as fatty degeneration, and vessels may appear as being telangiectatic, sinusoidal or with thickened walls. Cells typically appear elongated, with ovoid and regular nuclei. As this type of lesion dislocates the nerve of origin during growth, both argentic staining and immunohistochemical assays for neurofilaments are capable of demonstrating that the tumour does not contain any axons. Thrombosis, haemorrhages and calcifications may be evident, and there may be positivity for S100 protein, glial fibrillary acidic protein (GFAP) and Leu7. Generally speaking, schwannomas are benign and affect sensory nerves. There may also be variant forms, such as the melanocytic, the plexiform, the reticular and the 'cellular' forms; in the latter case, even with the presence of mitosis, nuclear polymorphism and a compact aspect without palisades, the prognosis is benign.

Malign schwannomas usually do not originate from benign ones, but originate primarily as malignant tumours, and are considered to be part of neurofibromatosis $(37,38)$. They recur after exeresis, and tend to increase their malignancy over time, being undistinguishable from malign neurofibromas and neurofibromatosis. They are classified within the so-called group of tumours termed malignant peripheral nervous system tumours (41). Differently from benign schwannomas, malign schwannomas appear as not always delimited nerve or eccentric masses. Histologically, malign schwannomas appear similarly to fibrosarcoma, being constituted by cells and elongated nuclei that are organised in fascicles. Cells may also form small vortexes and, occasionally, palisades (39). Mitotic events are frequent. The tumour invades adjacent structures, tending to become more malignant as time proceeds, leading to necrosis and polymorphic elements, with a reduction of the fibrillary component. Immunohistochemical analysis generally provides evidence of positivity for S100 protein $(37,38)$.

In conclusion, HyN schwannomas occur only rarely, with few cases previously described in literature. Management of the disease consists of the surgical excision of the lesion whenever possible, due to the potential negative effects that it may have on the delicate surrounding structures. IC lesions with secondary EC extensions appear to be the most frequent type of lesions. Isolated EC lesions are rather rare. Second-level imaging, especially MRI, represents a useful technique to preoperatively study the lesion and plan the best surgical approach. Recovery of the nerve function is generally good, although mild deficits may remain, depending on the location of the lesion and on the complexity of surgical excision.

\section{References}

1. Anastasi G, Capitani S, Carnazza ML, Cinti S, De Caro R, Donato RF, et al: Trattato di Anatomia Umana. Edi Ermes, Milano, 2010 (In Italian).

2. Lee MK, Sidell DR, Mendelsohn AH and Blackwell KE: Hypoglossal schwannoma masquerading as a carotid body tumor. Case Rep Otolaryngol 2012: 842761, 2012.

3. Basaran B, Polat B, Unsaler S, Ulusan M, Aslan I and Hafiz G: Parapharyngeal space tumours: The efficiency of a transcervical approach without mandibulotomy through review of 44 cases. Acta Otorhinolaryngol Ital 34: 310-316, 2014.

4. Loeb C and Favale E: Neurologia di Fazio-Loeb. Rome: SEU, 2003 
5. Joshi R: Learning from eponyms: Jose Verocay and Verocay bodies, Antoni A and B areas, Nils Antoni and Schwannomas. Indian Dermatol Online J 3: 215-219, 2012.

6. Verocay J: Zur Kenntnis der 'Neurofibrome'. Beitr Pathol Anat Allg Pathol: 1-69, 1910.

7. Ghali MG, Srinivasan VM, Jea A, Slopis JM and McCutcheon IE: Neurofibromas of the phrenic nerve: A case report and review of the literature. World Neurosurg 88: 237-242, 2016.

8. Antoni NR: Über Ruckenmarkstumoren und Neurofibrome. Bergmann, Miinchen, 1920 (In German).

9. Yu Z, Zhao G, Zhao Z, Li Y and Xie G: Giant recurrent dumbbell-shaped hypoglossal schwannoma in an elderly male: A case report. Oncol Lett 11: 283-286, 2016.

10. Aihara K and Morita A: Dumbbell-shaped hypoglossal schwannoma in an elderly woman: A clinical dilemma. Surg Neurol 63 : 526-528, 2005.

11. Baghel PS, Gupta A, Tripathi VD and Reddy DS: Hypoglossal schwannoma presenting as hemi-atrophy of the tongue. Neurology India 61: 324-325, 2013.

12. Durnford AJ, Harrisson SE, Ditchfield A and Shenouda E Spontaneous regression of a cystic hypoglossal schwannoma causing unilateral tongue atrophy. Br J Neurosurg 28: 133-134, 2014.

13. Garg N and Sampath S: Fatal delayed post-operative cerebral venous thrombosis after excision of hypoglossal nerve schwannoma. Acta Neurochir (Wien) 150: 605-609, 2008.

14. Inoue H, Nakagawa Y, Ikemura M, Usugi E, Kiyofuji Y and Nata M: Acute brainstem compression by intratumoral hemorrhages in an intracranial hypoglossal schwannoma. Leg Med (Tokyo) 15: 249-252, 2013.

15. Ishikawa M,Kusaka G, Takashima K, Kamochi H and Shinoda S: Intraoperative monitoring during surgery for hypoglossal schwannoma. J Clin Neurosci 17: 1053-1056, 2010.

16. Kabatas S, Cansever T, Yilmaz C, Demiralay E, Celebi S and Caner H: Giant craniocervical junction schwannoma involving the hypoglossal nerve: Case report. Turkish Neurosurg 20: 73-76, 2010.

17. Kuo LT, Huang AP, Kuo KT and Tseng HM: Extradural dumbbell schwannoma of the hypoglossal nerve: A case report with review of the literature. Surg Neurol 70 (Suppl 1): S1:34-39, 2008

18. Li WC, Hong XY, Wang LP, Ge PF, Fu SL and Luo YN: Large cystic hypoglossal schwannoma with fluid-fluid level: A case report. Skull Base 20: 193-197, 2010.

19. Mariniello G, Horvat A, Popovic M and Dolenc VV: Cellular dumbbell schwannoma of the hypoglossal nerve presenting without hypoglossal nerve palsy. Clin Neurol Neurosurg 102: 40-43, 2000.

20. Mathiesen T, Svensson M, Lundgren J, Kihlström L, Parisotto R and Bagger-Sjöbäck D: Hypoglossal schwannoma-successful reinnervation and functional recovery of the tongue following tumour removal and nerve grafting. Acta Neurochir (Wien) 151: 837-841, 2009

21. Ohba S, Inaba M, Kinoshita Y, Nakagawa T, Shimizu K and Murakami H: Hypoglossal nerve schwannoma with intratumoral hemorrhage. Brain Tumor Pathol 29: 43-46, 2012.

22. Rachinger J, Fellner FA and Trenkler J: Dumbbell-shaped hypoglossal schwannoma. A case report. Magn Reson Imaging 21: $155-158,2003$

23. Ram H, Agrawal SP, Husain N and Chakrabarti S: Hypoglossal schwannoma of parapharyngeal space: An unusual case report. J Maxillofac Oral Surg 14 (Suppl 1): S73-S76, 2015.

24. Ranta A, Winter WC and Login IS: Extracranial hypoglossal schwannoma. Neurology 60: E11, 2003.
25. Santarius T, Dakoji S, Afshari FT, Raymond FL, Firth HV, Fernandes HM and Garnett MR: Isolated hypoglossal schwannoma in a 9-year-old child. J Neurosurg Pediatr 10: 130-133, 2012.

26. Sato K, Shimizu S, Oka H, Nakahara K, Utsuki S and Fujii K: Usefulness of transcervical approach for surgical treatment of hypoglossal schwannoma with paraspinal extension: Case report. Surg Neurol 65: 397-401, 2006.

27. Suthar PP, Mistry KA, Rajan P, Ankit P and Mehta C: Isolated hypoglossal nerve schwannoma: An uncommon presentation of schwannoma. J Clin Diagn Res 9: TJ01-TJ02, 2015.

28. Wanjari SP, Wanjari PV, Parwani RN and Tekade SA: Unusually large quiescent ancient schwannoma of hypoglossal nerve. Indian J Dent Res 24: 768-771, 2013.

29. Behuria S, Rout TK and Pattanayak S: Diagnosis and management of schwannomas originating from the cervical vagus nerve. Ann R Coll Surg Engl 97: 92-97, 2015.

30. Fornaro R, Frascio M, Stabilini C, Ricci B, Mandolfino F, Picori E, Sticchi C, Boccardo C and Gianetta E: Excision of a schwannoma of the neck: Surgical technique. G Chir 27: 428-432, 2006 (In Italian).

31. Fornaro R, Canaletti M, Spaggiari P, Davini MD, Masuri M, Sticchi C, Moraglia E, Capellino M, Picori E and Terrizzi A: Report on a case of schwannoma of the neck: Clinical and therapeutic considerations. Chir Ital 57: 91-98, 2005.

32. Haastert-Talini K, Geuna S, Dahlin LB, Meyer C, Stenberg L, Freier T, Heimann C, Barwig C, Pinto LF, Raimondo S, et al: Chitosan tubes of varying degrees of acetylation for bridging peripheral nerve defects. Biomaterials 34: 9886-9904, 2013

33. Duda S, Dreyer L, Behrens P, Wienecke S, Chakradeo T, Glasmacher B and Haastert-Talini K: Outer electrospun polycaprolactone shell induces massive foreign body reaction and impairs axonal regeneration through 3D multichannel chitosan nerve guides. Biomed Res Int 2014: 835269, 2014.

34. Geuna S, Raimondo S, Fregnan F, Haastert-Talini K and Grothe C: In vitro models for peripheral nerve regeneration. Eur J Neurosci 43: 287-296, 2016.

35. Meyer C, Stenberg L, Gonzalez-Perez F, Wrobel S, Ronchi G, Udina E, Suganuma S, Geuna S, Navarro X, Dahlin LB, et al: Chitosan-film enhanced chitosan nerve guides for long-distance regeneration of peripheral nerves. Biomaterials 76: 33-51, 2016.

36. Meyer C, Wrobel S, Raimondo S, Rochkind S, Heimann C, Shahar A, Ziv-Polat O, Geuna S, Grothe C and Haastert-Talini K: Peripheral nerve regeneration through hydrogel-enriched chitosan conduits containing engineered schwann cells for drug delivery. Cell Transplant 25: 159-182, 2016.

37. Kumar V, Abbas AK, Fausto N and Aster JC: Robbins and Cotran Pathologic Basis of Disease. 9th edition. Elsevier, 2014

38. Mancardi GL, Tagliavini F and Vita G; Italian Association of Neurology: Treatments of Neuropathology. Contatto \& Archimedica, Torino, 2007 (In Italian).

39. Wippold FJ II, Lämmle M, Anatelli F, Lennerz J and Perry A: Neuropathology for the neuroradiologist: Palizades and pseudopalisades. AJNR Am J Neuroradiol 27: 2037-2041, 2006.

40. Wippold FJ II, Lubner M, Perrin RJ, Lammle M and Perry A: Neuropathology for the neuroradiologist: Antoni A and Antoni B tissue patterns. AJNR Am J Neuroradiol 28: 1633-1638, 2007.

41. Rodriguez FJ, Folpe AL, Giannini C and Perry A: Pathology of peripheral nerve sheath tumors: Diagnostic overview and update on selected diagnostic problems. Acta Neuropathol 123: 295-319, 2012. 